\title{
تأثير وزن الكتكوت بعمر يوم واحد على صفات النمو وقطعيات الذبيمة لفروج اللم ROSS
}

\author{
مازن ناصر علي
}

قسم الانتاج الحيو اني، كلية ناصر للعلوم الزر اعبة، جامعة عدن، اليمن الباحث الممثَل: مازن ناصر علي؛ بريد الكتروني: nzam25@gmail.com استلم في: 22 نوفمبر 2021 / قبل في: 21 ديسمبر 2021 / نشر في: 31 ديسمبر 2021

\begin{abstract}
المُأخّص
أجريت التجربة في حظيرة قسم الانتاج الحيو اني كلية ناصر للعلوم الزر اعية م/لحج خلال الفترة 2020/1/28 لغاية 2020/3/2م، استهدفت



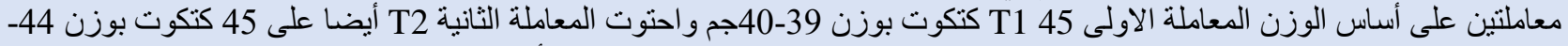

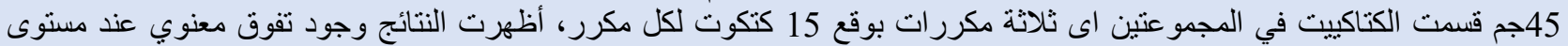

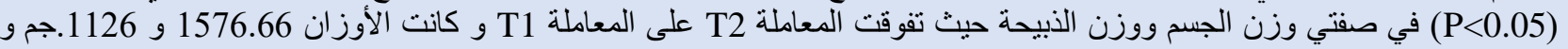

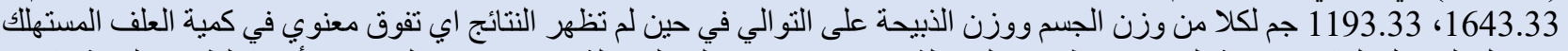

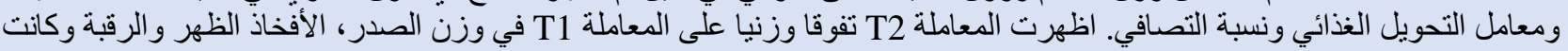

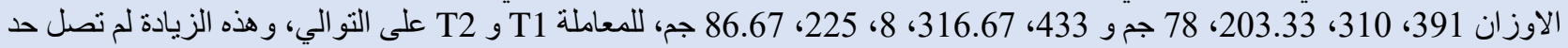

المعنوية.
\end{abstract}

\section{الكلمات المفتاحية: دجاج اللحم، الوزن بعمر يوم واحد، نمو.}

\section{3و مواد وطرق البيث:}

أجريت هذه التجربة في حظيرة قسم الانتاج الحيواني كلية ناصر للعلوم

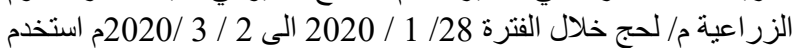

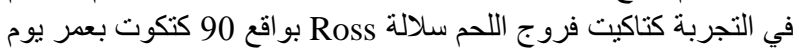

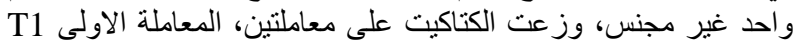

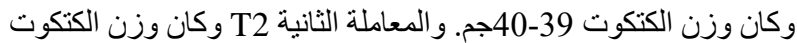

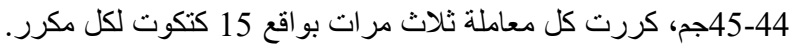

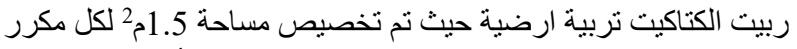

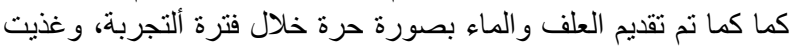

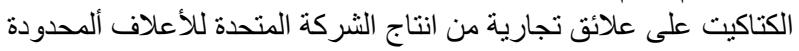

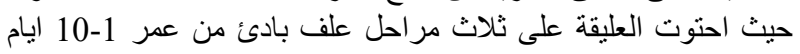

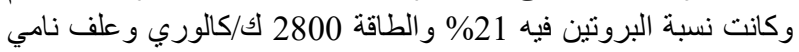

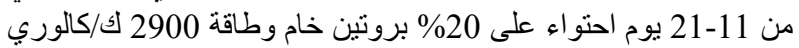

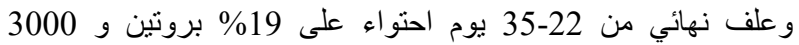

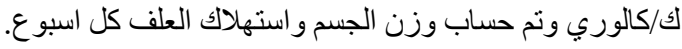
في نهاية التجربة تم اخذ ثلاثة طيور من كل مكرر وتم تصويمها عثر فئر

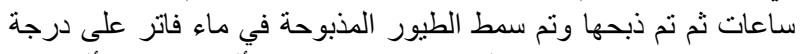

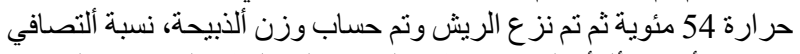

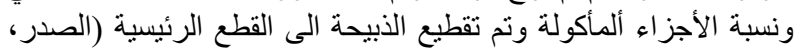

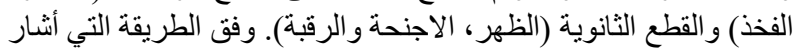

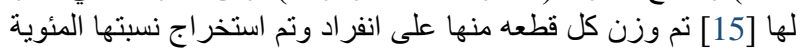

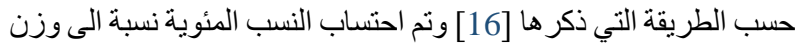
الذبيحة.

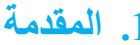

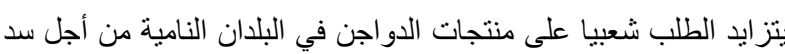

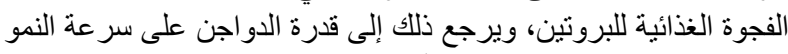

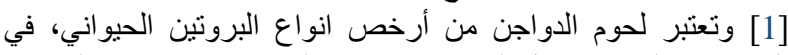

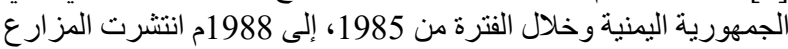

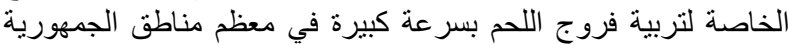

[2] اليمنية

أن وزن الكتاكيت في عمر يوم واحد له تأثير كبير على وزن الكتاكيت عند

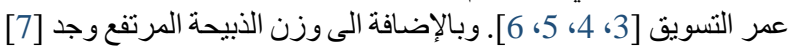

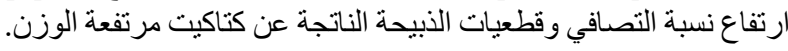

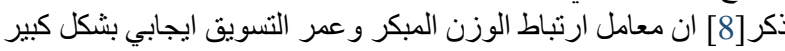
جدا في ألكتاكيت.

اوضح [9] ان الكتاكيت ذات الاوزان العالية تظهر زيادة كبيرة في وزن الكان

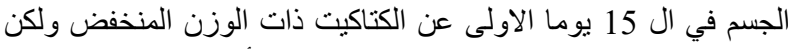

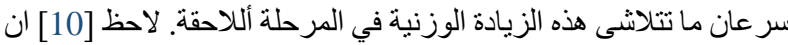

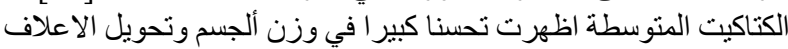

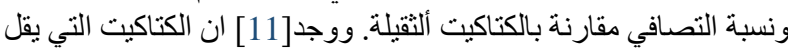

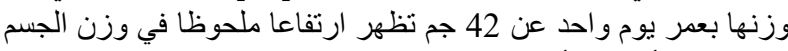

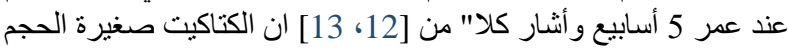

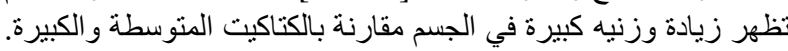
ولم يلاحظ [14] اي تأثير معنوي لوزن فئ الكتاكيت عند عمر التسويق.

$$
\text { 2. 2 الها ف من الار استة: }
$$

هدف هذا البحث الى در اسة تأثير وزن الكتاكيت بعمر يوم و احد على صفات النمو وقطعيات الذبيحة عند عمر التسويث. 
الاوزي T1 زيادة وزنيه على المعاملة T2 في وزن القلب و الطحال حيث بلغت

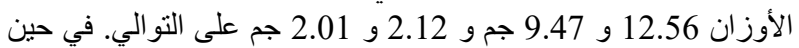

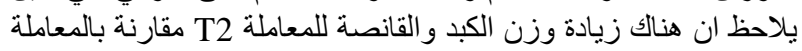

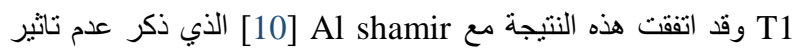

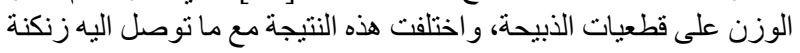

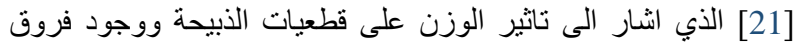

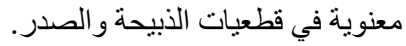
جدول (3): يبين تأثثر الوزن بعمر يوم واحد على وزن الكبد، القلب،

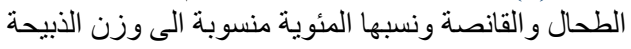

\begin{tabular}{|c|c|c|c|c|c|c|c|c|}
\hline \multicolumn{2}{|c|}{ لقانصة } & \multicolumn{2}{|c|}{ الطحال } & \multicolumn{2}{|c|}{ القلب } & \multicolumn{2}{|c|}{ الكبد } & الصفة \\
\hline$\%$ & جم & $\%$ & جم & $\%$ & جم & $\%$ & جم & المعاملة \\
\hline 1.75 & 27.97 & 0.13 & 2.12 & 0.79 & 12.56 & 2.13 & 33.95 & $\mathrm{~T} 1$ \\
\hline 1.73 & 28.22 & 0.12 & 2.01 & 0.58 & 9.47 & 2.23 & 36.23 & $\mathrm{~T} 2$ \\
\hline NS & NS & NS & NS & NS & NS & NS & NS & Lsd $\quad 0.05$ \\
\hline
\end{tabular}

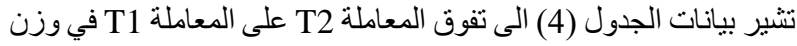

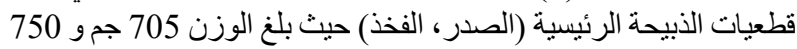

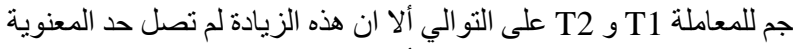

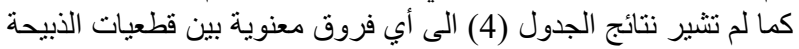

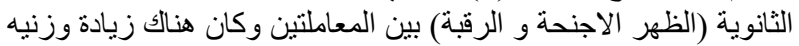

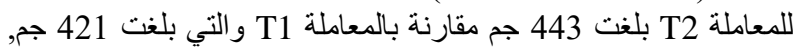

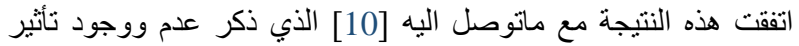

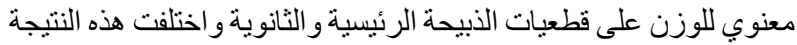

مع [7].

$$
\text { جدول (4): يبين تأثير الوزن بعمر يوم واحد على قطعيات الذبيحة الذيحة وزبية }
$$

\begin{tabular}{|c|c|c|c|c|}
\hline \multicolumn{2}{|c|}{ القطعيات الثانوية } & \multicolumn{2}{|c|}{ القطعيات الرئيسية } & الصفة \\
\hline$\%$ & جم & $\%$ & جم & المعاملة \\
\hline 37.41 & 421 & 62.58 & 705 & $\mathrm{~T} 1$ \\
\hline 37.14 & 443 & 62.86 & 750 & $\mathrm{~T} 2$ \\
\hline NS & NS & NS & NS & Lsd 0.05 \\
\hline
\end{tabular}

\section{5}

تشير نتائج الدر اسة الى ان الوزن المرتفع للكتاكيت اعطى اعلى وزن للجسم

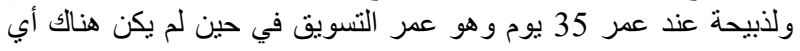

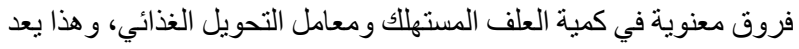
مؤشر على ان الكتاكيت مرتفعة الوزن بعمر يوم واحد هي الاكثر كفاءة في

$$
\text { 6. النمو. }
$$

1- نوصي بتربية الكتاكيت بوزن 44-45 جم من سلالة Ross.

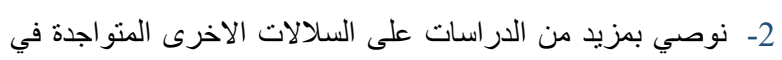

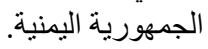

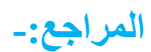

[1] A. M. King'ori, .Review of the factors influence egg fertility and hatchability in Poultry. International Journal of Poultry Science, 10: 483-492, 2011.

\section{التشيليل الاحصائي:}

حلت البينات باستخدام تقسيم القطاعات العشوائية الكاملة (R.C.B.D) Randomized complete Block Design بين المتوسطات أستعمل اختبار أقل فرق معنوي

significant Difference

\section{4. النتائج و المناقشُشة:}

تشير نتائج الجدول (1) إلى وجود فروق معنوي في صفة وزن الجسم

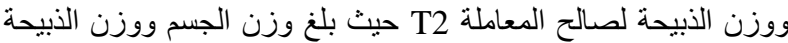



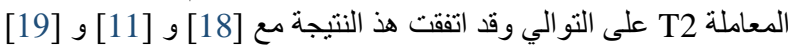

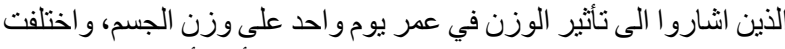

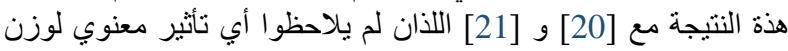

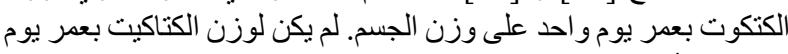

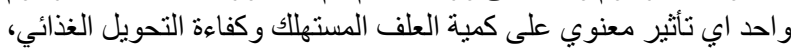

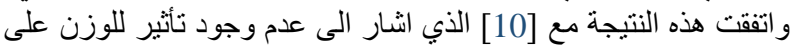

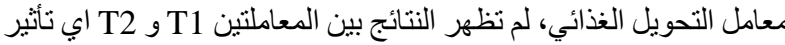
معنوي على نسبة التصافي.

\begin{tabular}{|c|c|c|c|c|c|}
\hline $\begin{array}{l}\text { التصبافية } \\
\%\end{array}$ & 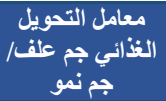 & المستهلك جم & وزن الذبيحة & هزن الجسم & وزن اليوم \\
\hline 71.48 & 1.559 & 2459.29 & $1126.67 \mathrm{~b}$ & $1576.66 \mathrm{~b}$ & $\mathrm{~T} 1$ \\
\hline 72.61 & 1.570 & 2570.00 & $1193.33 a$ & $1643.33 \mathrm{a}$ & $\mathrm{T} 2$ \\
\hline NS & NS & NS & 62.521 & 46.47 & Lsd 0.05 \\
\hline
\end{tabular}



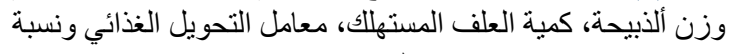
التصافي - (التي

أظهرت نتائج الجدول (2) عدم وجود فروق معنوية في قطعيات الذبيحة

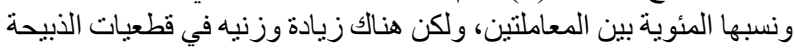

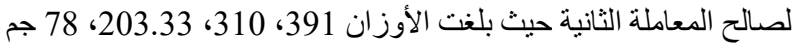

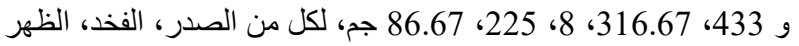

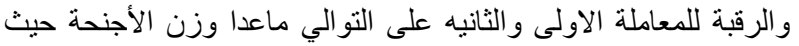

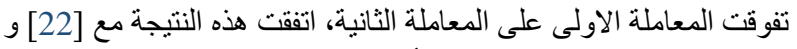



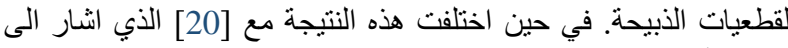

\begin{tabular}{|c|c|c|c|c|}
\hline L.S. D & $\mathrm{T} 2$ & T1 & \multicolumn{2}{|c|}{ المعاملة والصفة } \\
\hline NS & 433.33 & 391.67 & جم & \multirow{2}{*}{ الصدر } \\
\hline NS & 36.32 & 34.89 & $\%$ & \\
\hline NS & 316.67 & 310.00 & جم & \multirow{2}{*}{ الفذذ } \\
\hline NS & 26.54 & 27.58 & $\%$ & \\
\hline NS & 225.00 & 203.33 & جم & \multirow{2}{*}{ الظهر } \\
\hline NS & 18.86 & 18.08 & $\%$ & \\
\hline NS & 131.67 & 140.00 & جم & \multirow{2}{*}{ الجناح } \\
\hline NS & 11.02 & 12.46 & $\%$ & \\
\hline NS & 86.67 & 78.33 & جم & \multirow{2}{*}{ الرقبة } \\
\hline NS & 7.25 & 6.97 & $\%$ & \\
\hline
\end{tabular}

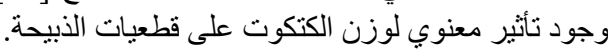

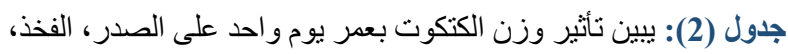

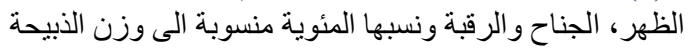

يبين جدول (3) عدم وجود فروق معنوية في صفة الوزن ونسبها المئوية

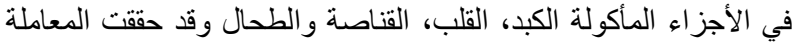


[12] M. Petek, A. Orman, S. Dikmen, and F. Alpay. Physicalchick parameters and effects on growth performance inbroiler. Arch. Anim .Breed., 53: 108-115, 2010.

[13] M. Michalczuk, M .Stêpiñska and M. £ukasiewicz. Effectof the initial body weight of Ross 308 chicken broilers onthe rate of growth.Annals. Warsaw Univ. Life Sci. SGGW,Anim Sci., 49: 121-125, 2011.

[14] R.J.R. Alzawbaii, Effect of Hatching Egg Weight on Fertility ,Hatchability and Subsequent Broiler Performance .M.Sc.college of Agriculture .University of Baghdad, 2010.

[15] United States Department of Agriculture (USDA).Poultry grading manual agriculture handbook number 31, Washington, 1998

$$
\begin{aligned}
& \text { [16] ط. ص. ف. المرسومي، تأثير إحلال الذرة البيضاء و الترتكلي محل }
\end{aligned}
$$

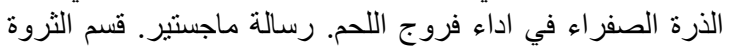

$$
\begin{aligned}
& \text { الحيو انية. كلية الزر اعة - جامعة بغداد، } 2000 .
\end{aligned}
$$

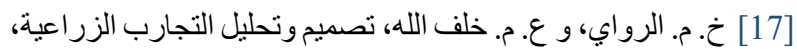

$$
\begin{aligned}
& \text { مؤسسة دار الكتب للطباعة والنشر، جامعة الموصلـ- العراق، } \\
& 1980
\end{aligned}
$$

[19] R.M.T. Khulel, and M. A. sabri. Effect of initial weight of chicks on performance of irqil chickens . plant Archives Vo(20) No (2) 5686 - 5690, 2020.

[20] R.S. Jiang, and N. Yang. Effect of day-old body weight on subsequent growth, carcass performances and level of growth-related hormones in quality meat-type chicken. Archiv fur Geflugelkunde, 71(2): 93-96, 2007.

$$
\begin{aligned}
& \text { [21] ب. س. ر. زنكنة، تقييم وزنين للفرخ في نسب القطعيات و التصافي }
\end{aligned}
$$

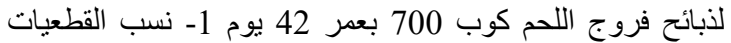

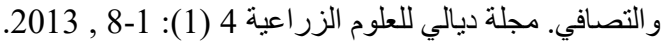

[22] R.M. Molenaar, M. Reijrink, R. Meijerhofand, H. Van. den Brand. Relationship between hatching length and weight on later production performance in broilers. Journal Poult. Sci., Vol,64:599-604, 2008
[2] O. A. Sallam, Abdul-Rahman, A. A, and H. A-m. Thbet. Evaluation of Broiler chicken productivity in some chicken commercial farm in the Republic of Yemen. Egyptian Journal of Agricultural Economics- Vol (19) No. (2): 615 - 622, 2009.

[3] K. Tona, O. Onagbesan, B. De Ketelaere, E. Decuypere \& V. Bruggeman, Effects of age of broiler breeders and egg storage on egg quality, hatchability, chick quality, chick weight and chick post-hatch growth to forty two days. The Journal of Applied Poultry Research, 13: 10-18, 2004.

$$
\begin{aligned}
& \text { 4] س. ع. ناجي، النمو التعويضي لمعالجة مشاكل السلالات الحديثة }
\end{aligned}
$$

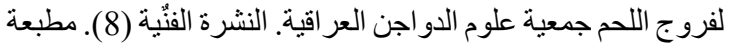

$$
\begin{aligned}
& \text { العصام، } 2006
\end{aligned}
$$

[5] M. Toghyani, M. Toghyani, A. Gheisari, G. Ghalamkari \&S. Eghbalsaied. Evaluation of cinnamon and garlic as antibiotic growth promoter substitutions on performance, immune responses, serum biochemical and haematological parameters in broiler chicks. Livestock Science-167 :138, 173.2011 .

[6] A. S. Mendes, SJ. Paixao, R. Restelatto, R. Reffatti , JC. Possenti , DJ. Moura, GMZ. Morello, \& de TMR. Carvalho. Effects of initial body weight and litter material on broiler production. Brazilian Journal of PoultryScience, 13: 165-170, 2011.

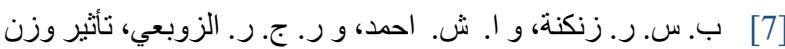

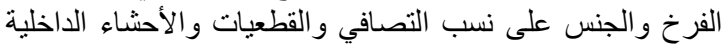
لذبائح فروج اللحم كوب

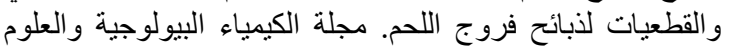

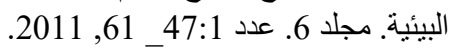

[8] K. G. Kim, E.S. Choi ,J.H. Kwon and S.H. Sohn TheEffect of Early Chick Weight on Market-Weight in KoreanNative Chickens .Korean Journal of PoultryScience, 44(4): 259-265, 2017.

[9] T.K. Patbandha, D.D .Garg, S. Marandi, D.G. Vaghamashi, S.S.Patil and H.H. Savsani. Effect of chick weight andmorphometric traits on growth performance of colouredbroiler chicken. J. Entomol. Zool. Stud., 5: 1278-1281, 2017.

[10] J.S. Al-shamire, Effect of day old chicks weight on productive performande of two broiler strains and their crossing. Iraqi journal of agricultural sciences, 47(5):1290-1297, 2016.

[11] A.M. Al-Nedawi, T.K .Aljanabi, S.M. Altaie, and F.R. AlSamarai (2019) Effect of sex and day-old weight on subsequentbody weight and body mass index in commercialbroilers. Adv. Anim .Vet. Sci., 7(1): 45-48, 2019. 


\section{RESEARCH ARTICLE}

EFFECT OF ONE-DAY-OLD CHICK'S WEIGHT ON GROWTH
CHARACTERISTICS AND CARCASS CUTS OF BROILERS CHICK'S
ROSS
Mazen Nasser Ali

Dept. of Animal Production, Collage of Nasser Science Agriculture, University of Aden, Yemen

*Corresponding author: Mazen Nasser Ali; E-mail: nzam25@gmail.com

Received: 22 November 2021 / Accepted: 21 December 2021 / Published online: 31 December 2021

\section{Abstract}

The experiment was conducted in the barn of the Department of Animal Production, Nasser College for Agricultural Sciences Lahij Governorate, during the period 28/1/2020 to 2/3/2020. The study targeted two weight categories of chicks. In the experiment, 90 unsexed one-day-old Ross chicks were used. These chicks were divided into. Two treatments based on weight. The first treatment T1, 45 chicks weighing 39-40 g, and the second treatment T2 also contained 45 chicks weighing 44-45 g. The chicks were divided into two groups that are, three replicates with 15 chicks for each replicate. T2 on treatment T1 and the weights were 15176.66, 1126.gm, 1643.33, 1193,33g for both body weight and carcass weight, respectively, while the results did not show any significant superiority in the amount of feed consumed, feed conversion factor, and dressing ratio. Treatment T2 showed a weight superiority over treatment T1 in the weight of the chest, thighs, back, and neck. The weights were 391, 310, 203.33, $78 \mathrm{~g}$ and 433, 316.67, 8, 225, $86.67 \mathrm{~g}$, for treatment $\mathrm{T} 1$ and $\mathrm{T} 2$ respectively, and this increase did not reach the significant level.

Keywords: Broiler chickens, One-day-old weight, Growth.

$$
\text { كيقية الاقتبّاس من هذا البعث: }
$$

م. ن. علي، " نأثير وزن الكتكوت بعمر يوم واحد على صفات النمو وقطعيات الذبيحة لفروج اللحم ROSS"، مجلَّة جامعة عدن الإلكترونيّة للعلوم الأساسيّة

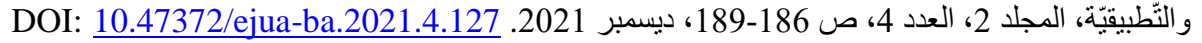

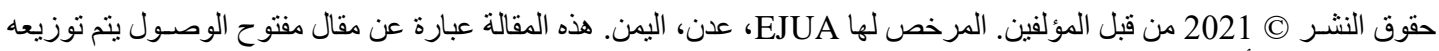

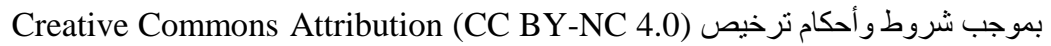

